\title{
La Crise des Établissements français des Indes
} (1768-1773)

The Financial Crisis in the French Establishments in India (1768-1773)

\section{Raphaël Malangin}

\section{(2) OpenEdition \\ 1 Journals}

\section{Édition électronique}

URL : https://journals.openedition.org/ahrf/13059

DOI : 10.4000/ahrf.13059

ISSN : 1952-403X

Éditeur :

Armand Colin, Société des études robespierristes

\section{Édition imprimée}

Date de publication : 1 mars 2014

Pagination : $69-88$

ISBN : 978-2-200-9083-2790-8

ISSN : 0003-4436

\section{Référence électronique}

Raphaël Malangin, «La Crise des Établissements français des Indes (1768-1773) », Annales historiques de la Révolution française [En ligne], 375 | janvier-mars 2014, mis en ligne le 01 juillet 2017, consulté le 05 juillet 2021. URL : http://journals.openedition.org/ahrf/13059; DOI : https://doi.org/10.4000/ahrf. 13059 


\title{
LA CRISE DES ÉTABLISSEMENTS FRANÇAIS DES INDES (1768-1773)
}

\author{
Raphaël MALANGIN
}

\begin{abstract}
D'une manière quasi générale, l'historiographie ancienne a imputé les difficultés que rencontrent les comptoirs français en Inde après 1765 au contrecoup de la guerre de Sept ans, à l'incompétence d'un personnel colonial prétendument corrompu et à l'incurie politique du gouvernement de Louis XV. L'objet de ce présent article est d'analyser les causes structurelles et conjoncturelles qui conduisent les comptoirs français à leur complète paralysie entre la fin 1768 et la fin 1773, d'envisager les stratégies qui ont été mises en place par le gouverneur Law de Lauriston et son conseil pour en assurer la survie, et d'essayer de mesurer la portée et les différentes conséquences, tant sociales, économiques que politiques, tant locales, régionales, qu'internationales, de cette crise profonde. Cette courte étude se propose donc de contribuer à faire émerger une image à la fois plus complexe et plus fidèle de la défaillance française en Inde à la fin du XVIII siècle.
\end{abstract}

Mots-clés : Inde, Compagnie des Indes, Pondichéry, comptoirs français, politique coloniale

Il y a, dans l'histoire de la présence française aux Indes au XVIII ${ }^{\mathrm{e}}$ siècle, une période assez peu connue, entre la crise de la Compagnie des Indes en 1768, la suspension du privilège en septembre 1769 et la mise en place effective d'une administration royale en 1773. Marguerite Labernadie, en 1936, avait souligné la rapidité de la reconstruction de Pondichéry, entièrement rasée en 1761 après le blocus dramatique de 
Coote ${ }^{1}$. Roger Glachant, en 1965, faisant le récit d'un désenchantement, avait mis l'accent sur l'impuissance politique, qu'il attribuait entièrement $\mathrm{au}$ « fossoyeur de nos espérances $»^{2}$, le gouverneur Jean Law de Lauriston, envoyé en Inde en 1764 pour reprendre possession des comptoirs indiens selon les termes de l'article 11 du désastreux traité de Paris de 1763, et qui fera cependant treize ans «d'un gouvernement général épineux $»^{3}$. Seul l'historien indien S.P. Sen, en 1971, avait mentionné en quelques mots les conditions qui prévalaient durant cette époque : «A period of acute financial distress $»^{4}$.

L'intérêt que nous portons désormais à l'histoire économique, sociale et financière des sociétés coloniales au XVIII ${ }^{\mathrm{e}}$ siècle, permet sans doute une réévaluation de cette crise, de ses causes et de ses conséquences à court et moyen termes, d'autant plus que le fonds « $\mathrm{C}^{2}$ Inde » conservé au Centre des Archives d'Outre-mer à Aix-en-Provence contient de nombreux documents éclairant utilement cette question. Certes, la crise de 1768-1773 n'a pas été l'unique crise de ce type dans les comptoirs français aux Indes orientales, mais elle en constitue bien le paroxysme, et elle permet de mieux juger des conditions du rétablissement français, du rôle effectif tenu par les acteurs, et de la place que l'Inde pouvait avoir dans la politique coloniale française à la fin du XVIII ${ }^{\mathrm{e}}$ siècle.

\section{La fabrique de l'impuissance : l'étranglement financier}

Force est de constater, en effet, que le simple effet du traité de 1763 n'a pas été l'unique cause de l'impuissance des Français en Inde. Le traité, s'il réduisait les possessions françaises à la portion congrue, celle qui prévalait avant l'année 1749, assurait théoriquement la pérennité du commerce. Il est donc nécessaire de recourir à d'autres explications, notamment financières, pour rendre compte du rapide dépérissement de la « présence française » en Inde après 1764.

Le caractère partiel et irrégulier des états de dépenses fournis par l'administration de l'Inde rend très aléatoire l'appréciation des besoins financiers au $\mathrm{XVIII}^{\mathrm{e}}$ siècle mais on est un peu mieux renseigné sur

(1) Marguerite LABERnADIE, Le vieux Pondichéry, 1673-1815, Histoire d'une ville coloniale, Paris, Imp. Moderne, 1936, p. 326.

(2) Roger Glachant, Les pluies et la poussière, Histoire de l'Inde des Français, Paris, Plon, 1965 , p. 154.

(3) AnOm, Cole 264, Dossier Law de Lauriston, Demandes du Sieur Law appuyées sur l'état de ses services [novembre 1780], Mémoire, $\mathrm{f}^{\circ} 66$. 1971, p. 70

(4) Siba Pada SEN, The French in India, 1763-1816, New Delhi, Munshiram Manoharlal, 
les recettes liées à l'exploitation du domaine, systématiquement mis en ferme ${ }^{5}$. Ces revenus étaient loin de suffire puisqu'il était nécessaire de faire parvenir à Pondichéry d'importants secours périodiques, mais trop souvent irréguliers surtout en temps de guerre, afin de financer les achats des effets de commerce et couvrir la majorité des dépenses ordinaires et l'intégralité des dépenses extraordinaires des comptoirs. Ces envois monétaires, essentiellement en argent, selon Philippe Haudrère, se font en piastres espagnoles que l'on se procurait à Cadix, exposant les vaisseaux de la Compagnie à des retards fréquents ${ }^{6}$. Cette anomalie manifeste aux théories mercantilistes constituait alors la principale fragilité du système, au point que Dupleix, en 1753, avait justifié sa politique expansionniste par des considérations financières. L'expansion était une nécessité car, disait-il, « toute Compagnie de commerce, quelle qu'elle soit, ne peut se soutenir par le simple bénéfice de son commerce, qu'il lui faut un revenu fixe et assuré, surtout lorsqu'elle a de grands établissements à soutenir $»^{7}$. La tension imposée au budget de la colonie était d'ailleurs une constante de l'histoire des établissements français en Inde depuis les origines. Du temps de Dupleix, les concessions allouées par Chanda Saheb ou Moussafer Jang d'immenses jagirs, ces seigneuries fiscales, avaient sans doute grandement contribué à soulager les inquiétudes d'argent et à procurer aux comptoirs une forme d'indépendance dont la Compagnie se méfiait irrémédiablement. Le renvoi de Dupleix en 1754, son remplacement par Godeheu puis par Duval de Leyrit et enfin la catastrophique campagne de Lally-Tollendal devaient marquer la fin de l'opulence et de l'affairisme français en Inde.

La situation que Jean Law de Lauriston trouva à son arrivée en 1764 accusa la fragilité financière des comptoirs : « Je n'ai jamais vu de destruction aussi acharnée que celle de Pondichéry, écrivait Law alors qu'il reprenait possession du comptoir en mai 1765, cela fait horreur, toutes les fortifications ont été ruinées par les mines, toutes les maisons rasées $»^{8}$. Le principal établissement en Inde, envahi par les herbes folles, livré aux chiens errants et aux serpents, laissait clairement augurer des investissements massifs qu'il faudrait y réaliser. Quant au domaine de la

(5) La Compagnie proscrit la mise en régie du domaine.

(6) Philippe HAUDRÈRE, La Compagnie française des Indes au XVIII siècle, 1719-1795, Paris, Librairie de l'Inde, 1987, p. 134.

(7) Cité par: Alfred MARTINEAU, La politique de Dupleix d'après sa lettre à Saunders du 18 février 1752, Pondichéry, Société de l'Histoire de l'Inde française, 1927, p. 99.

(8) ANOM, C ${ }^{2}$ 98, lettre de Law de Lauriston au ministre du 10 mai 1765 , f 220 . Le gouverneur Jean Law de Lauriston, ancien chef de la loge de Cassimbazar, était le neveu de John Law, l'homme de la banqueroute, et le père de Jacques Alexandre Law, le maréchal de France. 
Compagnie, amputé de ses plus vastes et lucratifs jagirs, les cinq circars, ou provinces, autour de Mazulipatnam, mais aussi les quatre aldées de Karikal, ou même les importants villages de Valdaour et de Vilenour autour de Pondichéry, ses revenus ne pouvaient guère soutenir l'activité commerciale et le fonctionnement des comptoirs : "Nous allons passer ici une vie bien désagréable - affirmait Law - [...] le cours des affaires de commerce sera souvent incertain moins par la mauvaise volonté des gens du pays que par les manœuvres sourdes des Anglais qui sont très piqués de nous revoir ${ }^{9}$. Enfin si l'actif de la Compagnie était fortement diminué en Inde, son passif, lui, n'avait cessé de s'accroître durant les trois ans d'absence. La Compagnie avait laissé de très nombreuses créances non soldées. Dès 1765 , la situation financière y était telle qu'elle perturbait considérablement l'affermage des droits de la Compagnie dont les représentants peinaient à trouver des fermiers solvables. Dans un mémoire de 1767, Law de Lauriston évaluait les revenus des établissements à 200000 livres, soit le cinquième de la somme nécessaire pour couvrir les dépenses totales ${ }^{10}$.

C'est dans ce contexte que la Compagnie inaugura, au début de 1767, un nouveau système de transfert de fonds vers l'Inde. Dans une situation financière de plus en plus précaire, la Compagnie trouva profitable de « dématérialiser » ses envois monétaires en Inde par le jeu de lettres de change sur Calcutta, en évitant ainsi les retards, le coût des opérations de change et des risques, tout en générant un intérêt. Ces lettres étaient gagées sur des particuliers anglais, mais parfois aussi hollandais, que leurs procureurs londoniens s'empressèrent d'accepter car elles permettaient le rapatriement discret des fortunes que la Compagnie anglaise interdisait formellement à ses employés. En Inde, les lettres de change à intérêt étaient payables à trois mois ou un an de vue. Au premier rang des débiteurs de la Compagnie française se trouvait donc la fine fleur du Conseil Suprême de Calcutta, Skinner, Bolt, les principaux gouverneurs, Vansittart, Verelst, et ses plus éminents militaires, tel Munro ${ }^{11}$. La Compagnie de France assortissait ces envois d'une défense absolue de se fournir en liquidité en Inde par des créances sur le trésor de la Compagnie.

Mais ce système montra ses limites dès la fin 1768 au moment de percevoir les premières traites. En septembre, à la surprise de Jean-Baptiste Chevalier, le directeur de Chandernagor, qui devait envoyer à Pondichéry

(9) Ibidem, lettre de Law au Duc de Choiseul, du 10 mai 1765, f 227.

(10) Siba Pada SEN, The French in India, op. cit., p. 70.

(11) ANOM, $C^{2} 120$, état des remises faites par la Compagnie en traites ...reçues en 1767, du $1^{\text {er }}$ octobre $1771, \mathrm{f}^{\circ} 247$. 
les sommes transférées, les Anglais se montrèrent incapables de fournir le moindre argent, et tout juste proposèrent-ils de l'or, ce qui induisait une perte de 16 pour cent, soit près d'un million sur les traites du Bengale ${ }^{12}$. Pour Chevalier, la raison en était évidente car le Bengale s'appauvrissait tous les jours : il en sort des quantités prodigieuses d'argent et il n'y en entre pas car «c'est le Bengale qui fournit toutes les dépenses immenses que la guerre de Madras contre Aidernaïck coûte aux Anglais. On leur a déjà fait passer pour 7 millions $\frac{1}{2}$ d'ici et ils en redemandent encore. C'est le Bengale aussi qui fournit l'argent nécessaire pour le commerce de la Compagnie anglaise à la Chine. Ce qui fait un objet de 7 à 8 millions par an $»^{13}$.

La situation ne tarda pas à s'aggraver en décembre 1767, car le Nabab du Bengale, Saiff ud-Dulah, fit poster des cipayes le long du Gange afin d'empêcher l'exportation de l'argent hors du Bengale, suivant l'ordre d'un Empereur alors prisonnier à Calcutta ${ }^{14}$. Tout cela était, selon Chevalier, « une machination inique tramée dans le noir cabinet de la politique anglaise $»^{15}$ :

«Ils ont appris que notre compagnie ayant fait passer tous ses fonds en lettres de change sur des particuliers de Calcutta, nous donnait ordre d'en envoyer partie pour le comptoir de Pondichéry, ils ont profité de cette occasion pour s'opposer à ce que nous puissions remplir cet objet, bien instruits que s'ils pouvaient y réussir, ils renverseraient notre commerce de la côte Coromandel et réduisait cette capitale de l'Inde française aux dernières extrémités $»^{16}$.

Ce complot anglais, au nom du Nabab et de l'Empereur qui exigeaient la visite des vaisseaux français par le Phozedar d'Ougly, l'officier du Nabab, Chevalier entendait bien le déjouer avec la plus grande fermeté, quitte à risquer l'ensemble du commerce français au Bengale, suspendu par le Nabab pendant plus de deux mois ${ }^{17}$. L'Empereur rétablit la liberté de commerce en mars 1769, mais Chevalier sentait bien que la solvabilité des lettres de change serait menacée tôt ou tard : «Et c'est ce qui va probablement arriver cette année, car la Compagnie nous annonce qu'elle

(12) ANOM, $C^{2}$ 99, lettre de Chevalier au Duc de Praslin du 6 septembre 1768, f ${ }^{\circ}$ 237-242.

(13) Ibidem.

(14) ANOM, $C^{2} 209$, lettre de Mamoud-Reza-Khan, régent du Royaume de Bengale, sd.(fin 1768), f ${ }^{\circ} 82$.

(15) ANOM, $C^{2} 210$, lettre de Chevalier au Duc de Praslin du 10 janvier 1769, f 98.

(16) Ibidem

(17) ANOM, $C^{2}$ 100, lettre de Chevalier au ministre de la Marine du 16 mars 1769, f f $^{\circ}$ 69-170. 
doit encore recommencer cette opération des lettres de change et les Anglais ont déclaré qu'ils ne les paieraient pas et qu'ils les laisseront protester. Fasse le génie tutélaire de la Compagnie qu'elle ait changé ses dispositions ${ }^{18}$.

Entre 1769 et 1771, on suit effectivement l'amoncellement des lettres de change non payées : rien que durant l'année 1769, sur un envoi total de près de 9 millions de livres, plus de 6 millions sont protestés soit par défaut d'acceptation, soit par défaut de paiement ${ }^{19}$. En février 1770, Law de Lauriston devait constater, amer : "Presque toutes les lettres de change que la Compagnie a envoyées sur les Anglais de l'Inde ont été protestées, de manière que nous sommes au moment de ne savoir ou prendre le $1^{\mathrm{er}}$ sol pour nos dépenses $»^{20}$. En août, arrivait à grands pas ce «moment fatal» où le trésor de la colonie serait complètement vide. Quant à l'espérance d'obtenir justice du Conseil de Calcutta, elle s'évanouissait devant les rumeurs d'une guerre européenne. En mars 1772, Chevalier réitérait ses demandes d'un jugement sans plus attendre ${ }^{21}$, et l'année suivante, le Conseil de Chandernagor constatait de nouveau le déni de justice le plus complet de la part des Anglais ${ }^{22}$.

Au début des années 1770, la situation financière des établissements paraissait donc très gravement compromise. De fait, les comptoirs ne reçurent aucun secours de France entre la fin 1767 et la fin 1771 et furent laissés entièrement à leur sort. L'arrivée de l'Abbé Terray au Contrôle des finances (1770-1774) et son intérim à la Marine (1770-1771) contribuèrent pour une part à cet abandon financier. Informé de la situation au moins depuis la fin 1769 , le ministère attendit avril 1771 pour expédier le vaisseau Le Gange avec les secours tant attendus ${ }^{23}$. Pour Chevalier, ce mystère ne pouvait s'expliquer que par de grossières erreurs dans les livres de comptes du Bengale, persuadant le ministre qu'il était « très riche alors qu'il était très pauvre $»^{24}$.

(18) Ibidem

(19) ANOM, C2 120, état des remises faites par la Compagnie en traites ...reçues en 1767, du $1^{\mathrm{er}}$ octobre $1771, \mathrm{f}^{\circ} 247$.

(20) ANOM, C ${ }^{2} 211$, lettre de Law à Mainon d'Invault du 24 février 1770, f ${ }^{\circ} 10$.

(21) ANOM, $C^{2} 122$, lettre de Chevalier et du conseil à Warren Hastings du 13 novembre $1772, \mathrm{f}^{\circ} 240$. $1773, \mathrm{f}^{\circ} 3$

(22) ANOM, $C^{2}$ 129, lettre du Conseil Supérieur au Conseil de Chandernagor du 21 février

(23) Les directeurs de la Compagnie des Indes affirment que ce sont les tensions entre l'Espagne et l'Angleterre qui en sont la cause. C $C^{2} 246$, lettre des directeurs au Conseil Supérieur de Pondichéry, du 16 mars $1771, \mathrm{f}^{\circ} 31$.

(24) ANOM, $C^{2} 120$, lettre de Chevalier au ministre de la Marine du 28 octobre $1771, \mathrm{f}^{\circ} 167$. 
En septembre 1771, Le Gange arriva en effet à Pondichéry mais il ne contenait que 500000 livres lorsque la dette se chiffrait déjà à plusieurs millions : «Le vaisseau Le Gange est bien arrivé - affirme Law - mais notre situation n'en est devenue que plus déplorable. Il nous restait du moins l'espérance, les expéditions venues par le vaisseau nous l'ont ôtée puisque nous voyons clairement qu'il ne nous est pas possible de nous soutenir n'y ayant l'avance des premiers secours que vous comptiez nous envoyer $»^{25}$. Il est cependant connu au ministère que cette somme ne suffira pas car il prévoyait de faire passer en Inde 700000 livres dans les mois suivants, montant notoirement insuffisant, mais, comme le rappelle alors un rédacteur du Bureau de l'Inde en août 1770 : « Sur un mémoire présenté au Roi par Monsieur le Contrôleur général dans son travail du 31 mars dernier, Sa Majesté a réduit à 1200 mille livres les dépenses de l'Inde qu'elle avait précédemment fixées à deux millions 400 mille livres $»^{26}$. La pénurie est donc bien le résultat d'un choix politique et non d'une erreur à laquelle les gouverneurs de l'Inde voulaient encore croire :

« Si par des raisons que j'ignore, l'administration veut toujours se servir de palliatifs, si lorsque nous disons noir, elle dit toujours blanc et cela à nous-mêmes, si lorsque nous marquons de concert avec messieurs de Chandernagor que nous n'avons aucun crédit, que messieurs les Anglais refusent de payer les lettres de change, l'administration au contraire nous répond et fait entendre au ministre que nous avons des fonds au Bengale, beaucoup de ressources, que les lettres de changes sont payées, si elle ne veut pas se fier à nous dans l'idée peut-être que pour nous mettre plus à notre aise nous faisons le mal beaucoup plus grand qu'il n'est, que nous reste-t-il à faire, sinon attendre une destruction qu'il ne nous est pas possible d'éviter $»^{27}$.

Pour parvenir à de telles réductions, un plan d'économies était nécessaire. Les instructions du ministre qui accompagnaient Le Gange, étaient drastiques. Chevalier, en octobre 1771, en était stupéfait : «Les dépêches arrivées par le vaisseau Le Gange ont jeté la consternation dans toute la colonie et nous ont causé d'autant plus d'embarras que l'exécution

(25) ANOM, $C^{2} 118$, lettre de Law à l'Abbé Terray du 21 septembre $1771, \mathrm{f}^{\circ} 247$.

(26) Ibidem, mémoire sur les fonds du département de l'Inde [août 1771], f 232-233.

(27) Ibid., lettre de Law au Conseil supérieur du 10 septembre 1771, f236. Law a manifestement tort de s'en prendre ainsi au Bureau de l'Inde qui avait averti le ministre: «On sait assez de quelle importance il est de ne point exposer les Établissements de l'Inde à manquer de secours et à s'anéantir d'une manière qui serait également préjudiciable et humiliante pour la nation ». cf. ANOM $\mathrm{C}^{2} 118$, mémoire sur les fonds du département de l'Inde, $\mathrm{f}^{\circ} 232$. 
des ordres qu'elles contiennent en est physiquement impossible $»^{28}$. Or les fonds du Gange ne pouvaient durer plus de quelques mois et il fallait tenir ainsi jusqu'à la mi-1772 car le vaisseau promis par Terray ne partirait qu'à l'automne. Aussi en 1772 et 1773 le trésor ne fut-il alimenté que par de modiques sommes arrivées par L'Étoile et L'Aquilon et toutes les dettes restèrent impayées. Seule la venue, le 2 octobre 1773, sur la flûte du roi $L a$ Fortune, du commissaire ordonnateur Foucault, chargé de mettre en place l'administration royale en Inde ${ }^{29}$, devait être le signe d'une normalisation progressive. Le trésor ne contenait plus alors que 324129 livres, qui, avec les 400000 qu'il apportait, devait suffire, selon Foucault, « jusqu'à l'arrivée du vaisseau qui devait partir de France en septembre dernier, en usant de beaucoup d'économie, et en réduisant les dépenses à l'indispensable $»^{30}$.

\section{Expédients et pis-aller : survivre à l'indifférence métropolitaine}

« Vous auriez vu, Monseigneur - écrivait Law au ministre en 1770 - l'état déplorable où nous sommes depuis dix mois que nous ne vivons, pour ainsi dire que par intrigues ; j' aurais même honte de vous détailler les moyens que la nécessité m'a obligé d'employer $»^{31}$. Dans cette situation, les Établissements français aux Indes orientales auraient dû s'effondrer d'eux-mêmes à l'automne 1770. Mais il n'en fut rien car il existait aux Indes des réseaux de finance ambigus qui sauvèrent in extremis les Français de la banqueroute.

Ces réseaux étaient connus de la Compagnie mais elle proscrivait formellement l'utilisation de lettres de change sur le trésorier de la Compagnie, afin de conserver la maîtrise de ses dépenses : " Je sais écrit l'un des directeurs à Law en mars 1768 - que c'est un moyen très commode de se procurer des fonds dans l'Inde, mais vous pouvez juger de la situation où se trouve la Compagnie à l'arrivée de ces lettres qui peuvent la constituer dans le plus grand embarras $»^{32}$. Elle préféra donc autoriser la vente des marchandises des magasins de Chandernagor et de Pondichéry qui devaient parfois constituer les cargaisons de retour, telles celle du Triton vendue en octobre 1770, mais, malgré toutes les facilités accordées aux

(28) ANOM, $C^{2} 120$, lettre de Chevalier aux directeurs de la Compagnie du 28 octobre 1771, $\mathrm{f}^{\circ} 145$.

(29) Si la cession des comptoirs au Roi eut effectivement lieu en 1770, elle ne fut pas suivie immédiatement d'une véritable réforme administrative.

(30) ANOM, E 190, lettre de Foucault au ministre de la Marine du 23 octobre $1773, \mathrm{f}^{\circ} 141$.

(31) ANOM, C ${ }^{2}$ 101, lettre de Law au Duc de Pralin du 18 août 1770, fo80-81.

(32) ANOM, $C^{2} 100$, citée dans l'extrait des délibérations du Conseil supérieur du 23 janvier $1769, f^{\circ} 103$. 
acheteurs, « la plupart de ces marchandises n'ont pu être vendues qu' au prix de facture, d'autres l'ont été à perte, et nous regarderions comme un succès si l'un dans l'autre toutes ces marchandises donnent un prix de facture de L'Orient $»^{33}$. On passa donc à la vente des effets de marine, puis l'on réfléchit à celle de plusieurs navires, mais ces expédients ne pouvaient suffire.

Aussi l'administration de l'Inde, de sa propre autorité, choisit-elle d'ignorer la défense d'emprunter afin de pouvoir expédier les vaisseaux de 1770 : « La Compagnie a crié beaucoup, à ce que j'ai ouï dire, sur les traites que nous avons ci-devant faites sur elle - écrivait Law en février 1770 mais aujourd'hui où serions-nous, bien plutôt, où en serait-elle si nous n'avions pas fait ces traites $»^{34}$. Mais ce ne fut qu'une solution passagère de plus : «Pour du crédit, il n'est plus nécessaire que la Compagnie nous défende d'emprunter, les Anglais y ont mis bon ordre, ils ont été depuis le rétablissement notre unique ressource et cela, il est vrai, tellement malgré eux que nous ne devons leur en avoir aucune obligation, mais aujourd'hui les ordres venus d'Europe ôtent toute espérance de côté-là » ${ }^{35}$.

Il ne restait donc plus qu'un moyen : faire passer le financement des établissements français pour celui des entreprises personnelles de Law et Chevalier auprès des créanciers, liant le sort de la nation française à la fortune d'une société que les gouverneurs avaient créée, à la fin 1767 , pour le commerce d'Inde en Inde et qui comptait de nombreux partenaires anglais ${ }^{36}$. Ces liaisons financières et commerciales étaient communes dans l'Inde car, affirmait Law au conseil assemblé, c'était une chose que tout le monde faisait pour son compte particulier, « en s'y prenant de manière que la chose ne fasse aucun éclat $»^{37}$. C'est pourtant contraint et forcé que Law en vint à cette solution, en août 1770 : «Il y a deux mois que nous cherchons de l'argent pour subvenir aux dépenses [...], on m'a offert plus de dix mille pagodes pour mon compte particulier si je donnais un billet à la grosse payable par mon procureur ; il a bien fallu en passer par là $»^{38}$. En effet, si Law venait à manquer à son engagement, les Anglais, expliquait-il

(33) ANOM, $\mathrm{C}^{2} 101$, lettre du Conseil supérieur aux directeurs de la Compagnie du 16 juin $1770, \mathrm{f}^{\circ} 165$.

(34) ANOM C $\mathrm{C}^{2} 211$, lettre de Law de Lauriston à Mainon d'Invault, le 24 février 1770, $\mathrm{f}^{\circ} 28-29$

(35) Ibidem, $\mathrm{f}^{\circ} 29$.

(36) ANOM C $\mathrm{C}^{2} 99$, lettre de Chevalier aux syndics et directeurs de la Compagnie du 30 décembre $1767, \mathrm{f}^{\circ} 85$.

(37) ANOM, $C^{2} 118$, lettre de Law au Conseil supérieur du 10 septembre 1771, fo250.

(38) ANOM, $C^{2} 101$, lettre de Law au Duc de Praslin du 18 août 1770, fo 82. 
l'année suivante, « peuvent parler haut, faire agir leur ambassadeur et me poursuivre en France ; de plus dans l'occasion dont il s'agit, ce ne sont point les fonds qui passent par nos mains, ainsi ils ne se trouvent pas dans le cas d'essuyer les moindres reproches de la part de leurs supérieurs $»^{39}$. Cette manipulation avait cependant son revers, et dès octobre Law, ne voyant aucun secours arriver, se plaignit au ministre :

«Il faut nécessairement qu'on ait persuadé à Monseigneur le Duc de Praslin et au Contrôleur général que Monsieur Chevalier et moi sommes deux crésus, ayant des fonds immenses et un crédit intarissable. Le vrai est cependant qu'aujourd'hui nous n'avons le sol ni l'un ni l'autre. Le peu que j'ai est à courir les mers, Dieu sait ce qu'ils deviendront. Le bien de Monsieur Chevalier court aussi les mers en partie, le reste est en Europe. A l'égard du crédit, je n'en ai pas aujourd'hui pour nos propres affaires, parce qu'on sait que je suis obligé d'employer à tout autre usage les fonds qu'on me donnerait $»^{40}$.

Le procédé fut cependant utilisé à plusieurs reprises entre la fin 1770 et la fin 1772, le plus souvent auprès de créanciers hollandais, il est vrai. Ce mode de financement, mêlant affaires particulières et publiques, intérêts de la Compagnie et intérêts privés, valut à Law et à Chevalier, à tort ou à raison, l'ire des esprits chagrins qui dénoncèrent le despotisme des gouverneurs ${ }^{41}$ : «Il n'est personne néanmoins qui à la première pensée n'envisage comme un très grand bien cette liaison universelle d'affaire des deux chefs de l'Inde - écrivait secrètement le conseiller Lagrenée de Mézière en octobre 1771 - mais je regarde cet accord comme l'effet le plus dangereux pour la prospérité d'une compagnie, parce qu'il ramène toutes les vues, toutes les actions de ces deux messieurs à ce qui a rapport uniquement à eux $\gg^{42}$.

Informé de la situation des finances à la fin 1770, le Duc de Praslin avait fait parvenir en Inde le plan d'une première réforme des employés, plan qui devait inquiéter Chevalier et dont il renvoyait l'exécution à la confirmation du ministre, car, affirmait-il, l'on n'a conservé au service que

(39) ANOM, C 2118 , lettre de Law au Duc de Praslin du 6 mai 1771, fo 224.

(40) ANOM, $C^{2}$ 101, lettre de Law au Duc de Choiseul du 20 octobre 1770, f 444.

(41) André Boyelleau, durant son intérim à Pondichéry en 1766, s'opposa vigoureusement à Law, resté au Bengale pour y redresser le commerce. Les conséquences politiques de cet épisode se firent sentir très longtemps. $1771, \mathrm{f}^{\circ} 279$.

(42) ANOM, $C^{2} 118$, lettre de Simon Lagrenée de Mézières à la Compagnie du 2 octobre 
des jeunes gens qui auraient besoin « de retourner à l'école d'où ils sont fraîchement sortis $»^{43}$.

En avril ou mai 1771, la rareté des fonds procurés par voie d'emprunt auprès des particuliers anglais ne permit pas d'éviter la suspension complète des salaires des employés de la Compagnie, conservés à la demi-solde depuis 1770 . C'est-à-dire la privation de la seule ressource pour vivre dans des comptoirs où toute l'activité des particuliers, hormis celle des chefs, s'était éteinte en 1761. À Pondichéry, un seul coup d'œil sur «l'air morne et abattu » de tous les habitants « suffit pour connaître toute la profondeur du mal qu'on ressent et fait apercevoir beaucoup plus qu'on ne peut exprimer $»^{44}$. Law en concluait qu'en mettant «toute cette Colonie à la presse, on n'en tirerait pas de quoi pourvoir à la durée d'un mois $»^{45}$. À Chandernagor, Chevalier dressait un tableau tout aussi noir du sort des employés : "Aucune ressource d'ailleurs, plus de crédit et plus de commerce en ce qu'il est devenu impraticable $»^{46}$.

La réforme Terray frappa donc ce microcosme fragile avec une rare violence en octobre 1771, imposant aux conseils de se séparer d'un employé sur deux. Chevalier en était stupéfait : "Plus de la moitié des employés se trouve compris dans la réforme, et ce qui m'a été encore plus sensible, messieurs, c'est que j'ai vu principalement de ce nombre tous ceux auxquels de tout temps j'ai pris le plus d'intérêt et que j'ai le plus particulièrement recommandés comme les meilleurs sujets $»^{47}$. Les employés réformés lui adressèrent une requête, se trouvant « continuellement dans la fâcheuse et dure nécessité de se refuser le pain et le vin de s'astreindre à vivre de riz et d'eau comme le dernier des noirs du pays et d'habiter ainsi qu'eux de mauvaises paillotes dont l'humidité ne défie pas moins leur santé que leur constitution $\gg^{48}$. Ils menaçaient de se retirer dans quelques colonies étrangères car, ajoutaient-ils : « Nous ne pouvons absolument nous promettre aucun secours de nos familles en Europe. Il est donc impossible de songer à y faire notre retour, puisqu'il est évident que nous y serions exposés à une misère plus grande encore que celle que nous éprouvons ici $\gg^{49}$.

(43) ANOM, $C^{2}$ 120, lettre de Chevalier au Duc de Praslin du 6 janvier 1771, fo $37-38$.

(44) ANOM C ${ }^{2} 101$, lettre de Law au Duc de Praslin du 18 août 1770, fo 87.

(45) Ibidem, $\mathrm{f}^{\circ} 81$.

(46) ANOM, $C^{2}$ 120, lettre de Chevalier au ministre de la Marine du 28 octobre 1771, fo 160.

(47) Ibidem, lettre de Chevalier aux directeurs de la Compagnie du 28 octobre 1771, f ${ }^{\circ} 145$.

(48) Ibid., copie de la requête [...] par messieurs les employés [...] du 14 octobre 1771, fo258.

(49) Ibid. 
Cette politique pouvait en outre coûter fort cher aux comptoirs. En effet, la réforme des employés supposait que ceux-ci soient payés intégralement de plus de dix-sept mois d'arriérés avant qu'ils ne quittent les comptoirs, et dans le laps de temps plus ou moins long entre leur réforme et leur départ, privés de toute subsistance, ils devenaient à charge de la colonie $^{50}$. Ainsi, tant par calcul que par humanité, les autorités de l'Inde abusèrent de la possibilité offerte par Terray de conserver à la demi-solde le personnel réformé qu'elles jugeraient nécessaire.

Il ne suffisait pas de réduire les dépenses, encore fallait-il augmenter des recettes que l'on ne pouvait trouver qu'auprès des Indiens. On mesure le durcissement de la politique de Law, habituellement assez débonnaire, à l'accroissement de l'inquiétude budgétaire. Dès la fin 1769, l'administration fit accélérer le recouvrement des créances du Bengale demandé par la Compagnie. Des ordres stricts furent ainsi donnés afin que les tisserands des harams, ou manufactures du Bengale, remboursent les avances qui leur avaient été faites avant 1757 et dont ils n'avaient jamais honoré les commandes. Mais, comme le constata Chevalier en 1771:

« Aussitôt que nous faisions arrêter un débiteur de la Compagnie dans l'instant celui-ci trouve protection assurée sous prétexte qu'il est tributaire ou fermier du gouvernement et c'est à ce titre que non seulement l'on ne souffre pas que nous l'obligions à payer ce qu'il nous doit mais même que l'on a poussé la violence jusqu'à aller à main armée enlever de nos loges ceux qui y étaient retenus à ce titre $»^{51}$.

Plus déshonorant encore aux yeux de Chevalier et de son conseil, au printemps 1772, les Anglais, au nom du Nabab, érigeaient un tribunal pour régler cette sempiternelle dispute et vérifier le bien fondé des créances françaises : «A la tête de ce tribunal, ils ont placé pour juge en dernier ressort un pion revêtu, leur serviteur à gages, à la décision duquel ils prétendent nous soumettre, en obligeant nos employés à comparaître devant lui et lui exhiber nos livres de négoce $»^{52}$. Chevalier savait d'ailleurs très bien que l'affaire des débiteurs ne pouvait véritablement se régler sans que soit terminé le remboursement de ses créanciers indiens de la Compagnie. À Chandernagor, le manque d'argent rendait la tâche de Panon et Nicolas,

(50) ANOM, $C^{2} 118$, lettre de Law à l'Abbé Terray du 21 septembre 1771, f 247.

(51) ANOM, $C^{2} 120$, lettre de Chevalier au ministre de la Marine du 28 octobre 1771, $\mathrm{f}^{\circ} 154-155$

(52) ANOM, $C^{2}$ 122, lettre du Conseil de Chandernagor au Conseil supérieur du 2 avril 1772, $\mathrm{f}^{\circ} 44$. 
chargés de la liquidation des créances de la Compagnie, de plus en plus difficile : "Ils commencent à nous menacer et nous craignons avec raison d'en être inquiétés dans peu si l'on ne trouve un moyen d'arrêter leurs cris et leurs clabauderies. Le seul remède qu'il y aurait serait de leur donner des acomptes ; mais où sont les fonds dont on puisse disposer à cet effet ? $»^{53}$. La situation de Pondichéry était depuis longtemps comparable : « Les gens du pays attachés à la nation ont été ruinés par la perte de Pondichéry et les malheurs qui ont suivi. Quand la confiance leur resterait, le pouvoir leur manque $»^{54}$.

Dans le désespoir complet, entre août 1770 et octobre 1771, on dut saisir la moindre aubaine pour financer le trésor de la colonie. Elle se présenta en juillet 1771 sous les traits de la veuve d'un Paléagar, un prince très riche, réfugiée avec ses beaux-frères dans la dépendance de Karikal afin d'échapper au Nabab qui les avait chassés de leurs terres. L'un des frères de ce Paléagar d'Orérapalayam s'était alors plaint à Law que sa belle-sœur monopolisait l'héritage familial et il lui promettait quarante à cinquante mille roupies s'il pouvait l'obliger à lui remettre ce qui lui revenait en partage :

« Cette femme étant entrée chez nous sans avoir donné ce qu'on nomme dans le pays un kaoul, c'est-à-dire une promesse de protection, affirmait Law [...]. Mon but était d'avoir de l'argent soit en emprunt soit autrement et je croyais la chose d'autant plus sûre que dans le cas où l'on aurait pu terminer la dispute entre cette femme et son beau-frère, je me flattais du moins de tirer quelque chose de cette femme pour droit de protection $\gg^{55}$.

Les négociations restant sans résultat, Law accorda sa confiance à un parent du conseiller Lecomte qui proposa de s'assurer de cette veuve par la force, mais le détachement fut trahi et deux cents paysans armés de sabres et de lances encerclèrent la vingtaine de cipayes. Des pourparlers se déroulèrent paisiblement jusqu'à l'incident :

«Un de nos soldats qui était saoul voulut trouver mauvais qu'un des paysans vint le regarder de trop près et sans ordre lui lâcha son coup de fusil qui le tua raide. Aussitôt les paysans attroupés tombent à coups de lance sur notre détachement [...], nos cipahis mal disciplinés prennent la fuite et

(53) Ibidem, $\mathrm{f}^{\circ} 43$.

(54) ANOM, $C^{2} 118$, lettre du Conseil supérieur au Contrôleur général du 25 octobre 1771, $\mathrm{f}^{\circ} 351$.

(55) Ibidem, lettre de Law à l'Abbé Terray du 20 octobre 1771, f²45-246. 
voilà la débandade. Ce ne fut qu'avec les plus grandes peines que l'officier vint à bout de gagner Karikal avec ses soldats dont les trois quarts étaient blessés et dont deux moururent quelques jours après. Il y eut aussi quelques paysans de tués et plusieurs blessés mais cette affaire n'a eu aucune suite.

Cette femme a décampé et s'est réfugiée dans le Tanjavour ${ }^{56}$.

En octobre, Law dut assumer la responsabilité de cette affaire, forfait honteux pour la nation et comble de l'impuissance imposée aux autorités de l'Inde. Il était clair que la crise des finances menait droit à l'impotence.

\section{Le maintien dans la paralysie et le discrédit}

Les conséquences de ces errements financiers, fruits d'un savant cocktail de vexations anglaises, d'incapacités, de divisions et d'ignorance des enjeux en France, dépassèrent de loin l'horizon des comptoirs et devaient avoir des conséquences dramatiques sur les objectifs et les moyens, tant militaires que diplomatiques, de la politique française en Inde à la fin du XVIII ${ }^{\mathrm{e}}$ siècle.

En Inde, pour restaurer la confiance dans la nation française, le moyen le plus sûr aurait été de rebâtir rapidement les fortifications, qui constituaient le meilleur symbole de puissance et d'investissement à long terme, le seul propre à rassurer la population, les créanciers et les princes indiens.

C'est un objectif prioritaire dès l'arrivée de Law à Pondichéry, qui avait fait sa traversée avec l'ingénieur Joseph Bourcet l'aîné en 1764. Ce dernier avait eu le temps d'observer les usages militaires et les moyens disponibles en Inde durant la campagne de Lally. Il en avait conclu, dans un mémoire adressé au ministre en 1762, au rétablissement des fortifications selon une méthode nouvelle qu'il avait vu appliquer à Calcutta et à Madras durant sa captivité, méthode qui permettrait, selon lui, un gain de temps et d'argent considérable ${ }^{57}$. Dans son projet, en effet, les revêtements s'appuyaient sur les talus selon un angle ouvert et consommaient jusqu'à deux fois moins de briques et de chaux. La fortification devait être réalisée en entier en moins de quatre ans.

Si Bourcet avait obtenu l'appui enthousiaste de Law de Lauriston, il subit l'hostilité de Boyelleau, lequel, critiquant son incompétence et le

(56) Ibid., Law se trompe, l'affaire aura des conséquences qui ne seront pas encore réglées en 1777.

(57) Voir notamment ANOM, 026 DFC 96 ${ }^{\text {ter }}$, précis d'un mémoire relatif à un projet pour le rétablissement des fortifications de Pondichéry par Bourcet [13 décembre 1762], $4 \mathrm{f}$. 
choix, selon lui malheureux, de ne pas relever sur les anciennes fondations, fit préparer par le conseil un contre-projet des plus irréalistes, justifiant ainsi la suspension des travaux jusqu'à l'arrivée des ordres d'Europe. A la fin 1768, rien n'avait donc été entrepris de plus sérieux qu'un simple retranchement. Par ailleurs, l'ingénieur éprouva les plus grandes difficultés à réunir les matériaux nécessaires et dut utiliser une majorité de briques de récupération des ruines de l'ancienne enceinte. Pour la chaux, écrit-il en mai 1767, « qui nous assurera encore que le Nabab à l'instigation des Anglais ne nous traversera pas, tirant la chaux de chez lui, lorsqu'on saura que les ordres de travailler aux fortifications seront arrivés $»^{58}$. C'est ainsi que les travaux commencèrent seulement en 1769 , malgré les dérèglements financiers au Bengale, car il s'agissait d'un choix politique très clair, que l'on rappelait en 1771:

« Rien de plus vrai, Monseigneur, que l'argent qui a été employé aux travaux devait l'être à payer les appointements [...]. Si nous avions la moindre ressource, nous ne pourrions nous empêcher de donner quelques acomptes. Le premier besoin est de vivre. Notre plus grand désir cependant est de nous voir fortifié : l'intérêt de notre conservation y est attaché $»^{59}$.

Mais les intrigues du parti Boyelleau bouleversèrent les efforts du conseil. Bourcet fut remplacé par un ingénieur du génie renommé, Jean Antoine Desclaisons, qui n' avait pourtant jamais travaillé en Inde. Lorsqu'il arriva à Pondichéry, en octobre 1769, il ne tarda pas à critiquer l'ouvrage, dont de graves défauts étaient effectivement visibles, et à exaspérer Law de ses nombreux mémoires. La reprise des travaux en 1770 devait rencontrer les habituelles difficultés auxquelles Desclaisons fit face avec beaucoup d'ardeur afin de démontrer la justesse de son point de vue. Ces quelques mois d'activité, au début 1770, devaient engloutir une quantité extraordinaire de matériaux pour réaliser un profil droit plus conventionnel. Law l'avait prédit quelque temps auparavant :

«Par le système de Monsieur Bourcet, nous avions trouvé le moyen de fortifier cette place d'une manière convenable selon moi, au climat du pays et à la nature du sol, dont nous avions d'ailleurs l'exemple devant nous. Cette dépense selon Monsieur Bourcet devait aller à deux millions,

(58) ANOM, 026 DFC 172, lettre de Bourcet aux syndics et directeurs de la Compagnie des Indes du 15 mai $1767,1 \mathrm{f}$.

(59) ANOM, $C^{2} 118$, lettre du Conseil supérieur de Pondichéry au Contrôleur général du 25 octobre $1771, \mathrm{f}^{\circ} 352$. 
mettons en trois. Il aurait tenu parole à peu de chose près. Aujourd'hui s'il faut abattre ce qui est fait, suivre exactement les plans envoyés d'Europe, fonder dans le fossé sans berne, la dépense ira peut-être au delà de sept millions $»^{60}$.

En août 1770, la crise des finances rendit inévitable l'arrêt des travaux. Desclaisons, dont la frustration avait été attisée par le camp Boyelleau, trouva la parade à cette inactivité forcée : il ne s'agissait rien de moins que de détruire les ouvrages de 1769 «parce que c'est un travail faible et nuisible, jeté dans travail solide qui en serait affaibli et même défiguré ${ }^{61}$ et d'en récupérer les briques. Cette prétention atterra le Conseil de Pondichéry pressé par les rumeurs de rupture entre l'Espagne et l'Angleterre. Elle eut sans doute un certain retentissement à Versailles, où l'on connaissait désormais la situation de Pondichéry, puisque Bourcet fut rappelé en avril 1771 pour venir terminer ses ouvrages. Quant aux travaux, ils ne reprirent qu'avec lenteur en janvier 1772, mais les àcoups financiers entravèrent des constructions et la ville était encore, en juillet 1776, « presque toute ouverte, et ajoutaient Law et l'ordonnateur Courcy, $[. .$.$] nous ne sommes au mieux que comme dans un camp assez$ mal retranché ${ }^{62}$.

La question des fortifications était indissociable de celle des forces armées. La nécessité d'envoyer des troupes en Inde, malgré le traité de Paris qui limitait la garnison de Chandernagor à 150 cipayes, tout le monde en convenait. C'était d'ailleurs la condition sine qua non des ambitieux projets militaires que Chevalier méditait seul ${ }^{63}$. À Pondichéry se trouvait le bataillon de l'Inde, renforcé en 1769 de 200 hommes, qui comprenait environ 600 Européens et le bataillon des cipayes, de même force $^{64}$. Plusieurs détachements du bataillon étaient stationnés à Mahé et Karikal. Mais cette troupe était notoirement insuffisante, et, entre 1767 et 1768 , Law réclamait avec insistance que l'on en renforçât l'artillerie ${ }^{65}$.

(60) ANOM, 026 DFC 239, extrait d'une lettre de Law à Mainon d'Inveau du 24 septembre $1769,5 \mathrm{f}$.

(61) ANOM, 026 DFC 342, mémoire de Desclaisons au Conseil Supérieur de Pondichéry du $1^{\text {er }}$ octobre $1770,4 \mathrm{f}$.

(62) ANOM C $\mathrm{C}^{2} 142$, lettre commune de Law de Lauriston et Courcy à Sartine, du 9 juillet $1776, \mathrm{f}^{\circ} 181$

(63) À ce propos, voir Jean DELOCHE, Jean-Baptiste Chevalier, le dernier champion de la cause française en Inde (1729-1789), Paris, EFEO/Les Indes savantes, 2003.

(64) Siba Pada SEN, The French in India, op. cit., p. 64.

(65) Ibidem, p. 63. 
Mais lorsque l'on n'était plus en mesure de payer les soldes, les troupes devenaient une menace. Tel fut le cas en août 1770, et l'on fit le choix de mettre les cipayes à la demi-solde tandis que les Européens percevraient une solde entière aussi longtemps que possible. En août 1771, la demi-solde n'était déjà plus versée depuis longtemps et Law s'en plaignait : «Les officiers, les employés, les cipayes ne sont plus payés, bientôt même je vais me voir dans l'impossibilité de payer les soldats $»^{66}$. À la fin de l'année, le conseil se trouvait face au dilemme d'avoir à mater des mutineries ou de voir déserter la garnison blanche ${ }^{67}$. En effet, la question des troupes «blanches » était étroitement liée à celle des troupes « noires » car, dans une place ouverte de toutes parts, «si nous n'avions pas des cipayes suffisamment pour garder les portes que nous avons établies, la garnison serait bientôt par désertion au tiers de ce qu'elle est $»^{68}$.

Aussi l'administration de l'Inde sentit toute la difficulté d'exécuter les ordres annonçant, la même année, la venue d'un renfort : "C'est dans un moment aussi critique qu'on me fait passer plus de six cents hommes dans une place dénuée de tout, ouverte de tous côtés. Voilà en soldats et cipayes ou invalides deux mille bouches au moins que j'ai à nourrir tant à cette côte qu'à la côte de Malabar, sans savoir où prendre le premier sol $»^{69}$. Et c'est avec la plus grande difficulté que Law parvint à les nourrir grâce au riz acheté à un conseiller hollandais de Negapatnam, dont le remboursement se fit fort péniblement en août 1771.

La réforme Terray, en septembre, n'eut guère d'effets positifs en ce qu'elle ne réglait aucunement la question de la paye et qu'elle réclamait le licenciement des cipayes afin d'alléger les dépenses. Inconséquence, selon Law, car « il nous est ordonné de réduire ceux-ci au nombre de 300, mais plus nous avons de soldats, plus nous avons besoin de cipayes pour empêcher la désertion. Il est bien triste qu'on nous ait fait passer tant de monde ; l'argent employé à leur paie aurait servi aux travaux qui se seraient trouvés bien plus avancés $»^{70}$.

Lorsque l'affaire de Tanjore éclata en 1771, Chevalier estimait que l'intérêt de la nation serait de seconder le Raja, «mais où en sont

(66) ANOM C² 118, lettre de Law à l'Abbé Terray du 21 septembre 1771, fo 247.

(67) À Mahé, Pondichéry et Karikal plusieurs mutineries sont attestées entre 1769 et 1771, notamment en rapport avec le défaut de paiement de la solde. Elles sont étouffées par l'administration qui menace d'utiliser ses troupes blanches.

(68) ANOM C2 101, lettre de Law au Duc de Pralin du 18 août 1770, fo66.

(69) Ibidem.

(70) ANOM, $C^{2} 118$, lettre de Law au Conseil supérieur du 10 septembre 1771, fo242. 
les moyens ? Nous n'avons point de troupes $\gg^{71}$. Mais Law faisait peu de cas d'une politique indienne incertaine lorsque la première nécessité commandait de trouver des fonds :

« On vous parle, Monseigneur, d'Aider ali khan, peut-être aussi du Roi de Tanjavour. On a vanté les secours qu'on pourra lever du premier, on aura peut-être poussé les choses jusqu'à se flatter d'écraser bientôt les Anglais par le moyen de ce prétendu conquérant. Tout cela n'est que fumée [...]. Aider Ali khan dans son temps le plus brillant ne nous aurait pas sauvés si les Anglais avaient voulu nous prendre $»^{72}$.

Il est vrai que la vénalité des princes indiens est bien connue et que leur fidélité va souvent au plus offrant, ce que même Chevalier devait reconnaître : «Combien l'on a à redouter des intrigues des Anglais et de leur argent. Ce sont des armes dangereuses qu'ils n'ont jamais employées sans succès vis-à-vis des princes du pays $\gg^{73}$. Il n'est donc guère étonnant que ni Law ni Chevalier ne purent compter sur les nababs pour se tirer de leurs embarras financiers. D'après Chevalier, Mahamet Ali, la marionnette des Anglais de Madras, fut le seul à compatir aux malheurs français : «Je prêterais volontiers à votre nation la somme dont vous me parlez - aurait-il déclaré à Guyonnet, son chirurgien français - ce ne serait pour moi qu'une bagatelle. Je sais d'un autre côté que Monsieur Law est un homme discret et à qui je puis me fier, mais je crains les livres de la Compagnie et encore plus les Anglais qui ne manqueront pas d'en avoir connaissance $\gg^{74}$. À la fin des tensions entre l'Angleterre et l'Espagne, Chevalier faisait le compte des occasions perdues :

« Dans la position où nous nous trouvons aujourd'hui, la nation a achevé de perdre le reste de crédit et de considération qu'elle conservait encore [...], vous ne pourriez croire, Monseigneur, tout le mauvais effet qu'a fait aux yeux des peuples cette réforme des employés [...]. L'on en a inféré que notre gouvernement est, sans doute, réduit à de très grands expédients pour être obligé d'avoir recours à ceux-là et qu'à bien plus forte raison, il serait entièrement hors de son pouvoir de toutes autres hautes entreprises $»^{75}$.

(71) ANOM, $C^{2}$ 120, lettre de Chevalier à l'Abbé Terray du 30 octobre 1771, f 202.

(72) ANOM, $C^{2} 118$, lettre de Law à l'Abbé Terray du 21 septembre 1771, fo248.

(73) ANOM, $C^{2} 120$, lettre de Chevalier à l'Abbé Terray du 30 octobre 1771, fo 203.

(74) Ibidem, lettre de Chevalier au ministre de la Marine du 28 octobre 1771, fo 196 . Guyonnet, ancien chirurgien de marine de la Compagnie des Indes, travaillait aussi pour le compte de Law.

(75) Ibidem, $\mathrm{f}^{\circ} 166$. 
Que restait-il alors de l'influence de Pondichéry et de ses comptoirs en Inde ? Peut-être un vaste réseau de Français au service des princes indiens, éparpillés en Inde depuis 1761. Les plus utiles à la France, selon les autorités, étaient Madec à Agra, Gentil auprès de Sujah-ud-dola à Patna, et, au Sud, Hugel, au service d'Aider Ali Khan. Les forces militaires dont ils disposaient personnellement étaient loin d'être négligeables. Cependant le rôle de ces entrepreneurs de guerre restait ambigu car, si Chevalier voulait croire qu'elle se révélerait cruciale le moment venu, leur présence motiva une importante désertion dans les troupes et il était rare que ces mercenaires soient des éléments de valeur. Chevalier ne voit ainsi chez Sujah-ud-dola «pas plus d'une douzaine de Français dans son service et à l'exception de deux ou trois, il serait à souhaiter qu'ils n'y fussent pas $»^{76}$. Dans cet interlope diplomatique et politique s'engouffraient donc des aventuriers de tout poil. L'aventure de De Braux, en 1770, en est la parfaite illustration. Cet ancien militaire de l'Inde, qu'une déchéance avait renvoyé au Bengale dans l'espoir d'une fortune rapide, était parvenu à se faire passer auprès de Sujah-ud-dola pour le négociateur du Duc de Choiseul et auprès de l'Abbé Terray pour l'envoyé du nabab. Lorsqu'il fut arrêté à Chandernagor, il était porteur de présents envoyés par Terray et d'un traité d'alliance dont les autorités n'avaient aucune connaissance. L'affaire était devenue publique et « tous nos jeunes gens ne le saluent partout que sous le titre de Monsieur l'Ambassadeur - déclarait Chevalier. Les Anglais n'en tarderont pas à être instruits, s'ils ne le sont déjà, ce qui augmentera de plus en plus leur vigilance et leur attention à observer de plus près encore toutes nos démarches $»^{77}$.

Telle est peut-être là la conséquence ultime d'une impuissance qui ne doit finalement pas grand-chose au hasard. Le renoncement à l'Inde fut peut-être un renoncement aux faits, et Law de peindre la politique indienne de la France avec clairvoyance :

«On nous prendrait volontiers pour des enfants entourés de leurs joujoux qu'ils agitent l'un après l'autre sans dessein, sans savoir ce qu'ils font. Nous employons vingt machines qui n'ayant aucun rapport entre elles, ou plutôt qui n'en ayant que de faux ne peuvent avoir qu'un mauvais effet, l'une détruit en partie ce que l'autre a fait. Cela vient encore de ce qu'on n'a pas grande confiance en ceux qui commandent dans l'Inde [...]. On aime bien mieux raisonner avec un homme d'esprit qui ayant ses 
vues particulières dont on ne s'aperçoit pas et n'ayant qu'une connaissance superficielle, ou même très fausse de l'Inde présentera hardiment les choses du côté agréable $»^{78}$.

Il est donc possible de tirer quelques leçons de cette crise. La première est sans doute que cette « zone grise » de l'histoire des Établissements est d'une importance majeure puisqu'il s'agit très clairement, pour la présence française en Inde, d'une question de vie ou de mort. La seconde, c'est qu'il ne sert à rien d'accuser Law et les principaux acteurs français en Inde d'avoir été les fossoyeurs de nos espérances, les sources qui montrent le contraire abondent. L'on devrait sans doute même les remercier d'avoir sauvé les Établissements d'un anéantissement total et honteux en temps de paix. L'étouffement est avant tout le fruit d'une crise à la fois conjoncturelle et structurelle et du manque de volonté de Versailles qui fait de l'Inde l'une des « variables d'ajustement » de sa politique financière. La troisième, c'est l'exceptionnelle résilience de ces comptoirs qui se refusent à disparaître. Elle montre un phénomène peu connu, digne de l'attention des historiens, l'étroite intrication des réseaux d'argent anglais et français en Inde, ainsi que le mélange caractéristique des affaires particulières et publiques ${ }^{79}$.

\author{
Raphaël MALANGIN \\ Professeur au Lycée français de Pondichéry \\ Université de Nantes \\ malangin.raphael@gmail.com
}

(78) ANOM, C² 118, lettre de Law à l'Abbé Terray du 13 octobre 1771, fo 252.

(79) A ce propos voir aussi passim: Arvind SINHA, The Politics of Trade : Anglo-French Commerce on the Coromandel Coast, 1763-1793, New Delhi, Manohar Publishers \& Distributors, 2002. 